\title{
Comparing Motion-based Versus Controller-based Pseudo-haptic Weight Sensations in VR
}

\author{
Yutaro Hirao* \\ Tuukka M. Takala \\ Anatole Lécuyer \\ Department of Intermedia Art and Science \\ JSPS International Research Fellow \\ Inria, Univ. Rennes, IRISA, CNRS \\ Waseda University \\ Waseda University
}

\begin{abstract}
This work examines whether pseudo-haptic experiences can be achieved using a game controller without motion tracking. For this purpose, we implemented a virtual hand manipulation method that uses the controller's analog stick. We compared the method's pseudo-haptic experience to that of the conventional approach of using a hand-held motion controller. The results suggest that our analog stick manipulation can present pseudo-weight sensations in a similar way to the conventional approach. This means that interaction designers and users can also choose to utilize analog stick manipulation for pseudo-haptic experiences, as an alternative to motion controllers.
\end{abstract}

General Keywords: Virtual reality, perception, design.

Author's Keywords: Pseudo-haptics, cross-modal interaction, human information processing, embodiment, virtual reality

Index Terms: [Computer graphics]: Graphics systems and interfaces---Perception; [Computer graphics]: Graphics systems and interfaces---virtual reality.

\section{INTRODUCTION}

Virtual Reality (VR) applications routinely utilize motion tracking technologies as much as possible. Tracking of the user's hands and head is commonplace, and there is a push for tracking the rest of the body: fingers, torso, and legs. In this paper, we go into the opposite direction and explore the possibilities of traditional game controllers without motion tracking. Specifically, we are interested in if it is possible to induce pseudo-haptic experiences using such controllers. To this end, we compare a motion controller's (motionbased) and a traditional controller's (controller-based) pseudohaptic weight sensations in VR.

We motivate our research by considering those users, who can use an analog stick but are unable to use motion-based manipulation: firstly, users with disabilities that prevent performing the required motions. Secondly, users who do not have the required VR systems and motion controllers, but who still want to utilize "VR only" applications such as Half-Life: Alyx, whose announcement angered a number of gamers without VR equipment.

If pseudo-haptic experiences can be achieved via controllerbased manipulation, this presents an additional option for VR interaction designers. This could result in added diversity both in users and user interfaces of various VR applications in fields such as entertainment, healthcare, education, and training.

\section{Related Work}

Different ways of presenting tactile and force feedback has been studied for a long time in the field of haptics research [1]. Such

\footnotetext{
* email: yutaro-hirao@suou.waseda.jp
}

feedback can be used to enhance interaction with virtual objects, for which various approaches have been implemented. Force feedback can be presented for example by grounded force feedback displays such as PHANToM [2] and SPIDAR [3], which mechanically reproduce the appropriate reaction force with relatively high accuracy. However, grounded force feedback displays limit the range of users' motion and suffer from poor portability. Recently, various ungrounded interfaces of presenting weight sensations in VR have been intensively studied in a field of HCI, such as a controller whose center of gravity can be shifted [47], utilizing air drag [7], and utilizing propeller-induced propulsive force [8]. These interfaces successfully produce a sense of weight or inertia. However, they generate unintended noise or haptic feedback such as vibration or inertia. Moreover, maintenance frequency is high as the interfaces are complicated.

An alternative approach is to utilize human cognitive characteristics for inducing haptic illusions. There are various such approaches for presenting a sense of weight, including applying force to the skin of the fingers in the shear direction [9], compressing users' wrist [10], and using a device that causes asymmetric vibration to utilize nonlinearity of human senses [11].

Another area that is intensively studied, is pseudo-haptics [1217], which refers to methods that rely on cross-modal integration. These approaches require relatively simple hardware and are compatible with Head-Mounted Displays (HMD) that can present rich audio-visual information. Compared to other approaches, pseudo-haptics allow a wider range of user motion, combined with fewer hardware problems such as weak motor output, heavy device weight, and low spatio-temporal resolution. For instance, in the research of Dominjon et al. [13], Taima et al. [14], Rietzler et al. [15], Samad et al. [16] and Hirao et al. [17], a sense of weight is presented by changing the amount of movement between virtual hand and actual hand.

These pseudo-haptic approaches are mainly achieved with a motion tracking system. Our research question is whether pseudohaptic experience can be achieved without motion tracking, and only by relying on traditional controller manipulation. We compare motion-based versus controller-based pseudo-haptic weight sensations in VR. Lin et al. have examined the difference of finger tracking (motion-based) manipulation versus VR controller-based manipulation on the virtual hand illusion in VR [18]. However, to the best of our knowledge, there is no previous research evaluating controller-based pseudo-haptic weight sensations in VR.

According to the model of Pusch and Lecuyer that explains the mechanism of pseudo-haptic experience [19], each sensory information such as vision, audition and body state (e.g. proprioception and touch) is weighted and integrated in accordance with Bayesian integration theory. This means that we need information about body state as well as vision or audition to feel pseudo-haptics.

However, when we use a game controller, we usually do not move our bodies except for our fingers. Nonetheless, it is conceivable that there are ways to make us feel as if our body is moving in those situations. Ma and Hommel have experimentally confirmed that a sense of body ownership was induced for a virtual balloon that changed its size, and also for a virtual square that changed its size and color synchronously with hand's motion [20]. According to this result, they concluded that non-corporeal events 
can be perceived as body parts if their changes are systematically related to one's actions. This supports the idea that we could feel as if our body is moving even when manipulating a virtual body via a game controller. Other studies have shown that passive haptic feedback during the interaction with virtual objects enhances the virtual environment and experiences in VR $[21,22]$. Therefore, we consider that the passive haptic feedback from a game controller could also enhance the pseudo-haptic experience. From the above, we hypothesize that users can feel pseudo-haptics via the controller-based manipulation of a virtual body.

\section{FIRST EXPERIMENT}

We conducted our first experiment to explore controller-based pseudo-haptics by comparing two manipulation methods for the task of pulling a virtual object in VR (Fig. 1).

\subsection{Equipment and stimuli}

Participants put on a VR HMD (Oculus Rift CV1) and held a VR controller (Oculus Touch) with their right hand. Figure 2 shows the experimental environment in VR. A virtual box with a handle was presented in front of the participants.

In the motion-based manipulation, the position and rotation of a right-hand model followed those of the VR controller. In this condition, participants extended their actual hand to the handle of the virtual box, pressed the controller's front trigger to grab it, and pulled their actual hand towards their body to drag the virtual box (Fig. 1; Motion-Based Manipulation).

In the controller-based manipulation condition, Oculus Touch was used as a traditional controller without motion tracking, and the hand model stayed in the air, regardless of the position of the actual hand that is holding the controller. Participants were able to move the hand model in the range between the start and the end point (Fig. 2) by tilting a controller's analog stick forward or backward. The top velocity for the hand was $2 \mathrm{~m} / \mathrm{s}$ in the utmost tilt

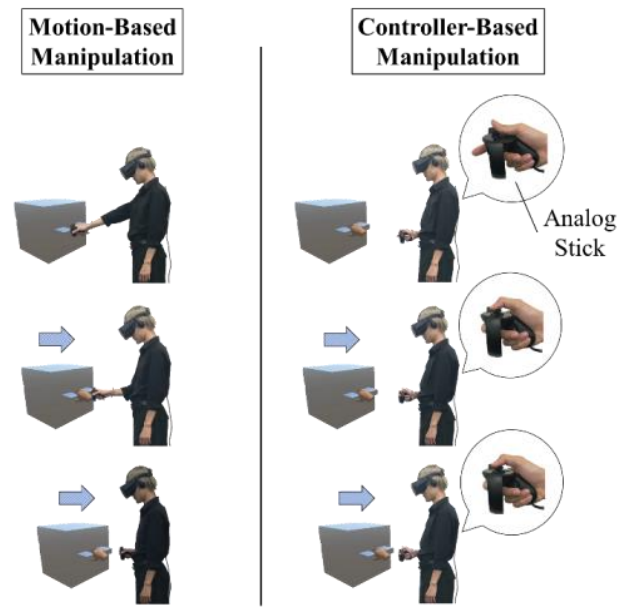

Figure 1: Motion-based and controller-based manipulation.

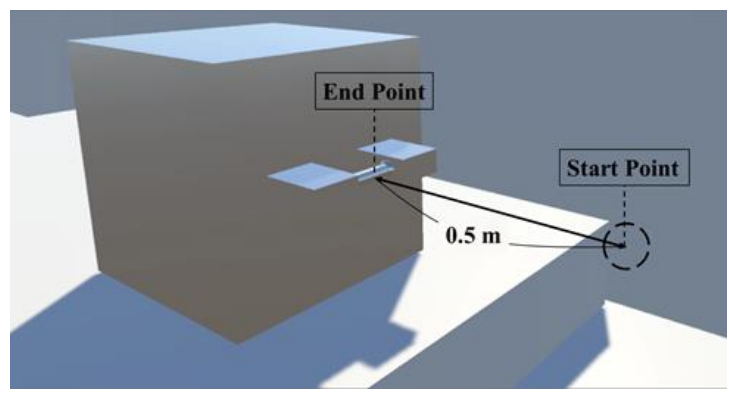

Figure 2: Experimental environment in VR. angle of the stick. This top velocity was determined by a pilot experiment, where two participants (including the author) tuned the velocity so that it seemed to be natural. During operation the hand velocity was interpolated from 0 to top velocity, depending on how the stick was positioned between the "dead zone" and the utmost tilt angle. The experimental task in this condition was for the participants to tilt the controller's analog stick forward to move the hand model to the handle position, press the trigger to grasp the handle, and then tilt the analog stick back to pull the virtual box towards them (Fig. 1., Controller-Based Manipulation). In both manipulation conditions, the handle (and the virtual box) moved from the end point to the start point (Fig. 2).

The technique of Hirao et al. [17] was used to induce pseudohaptic weight while the participants dragged the virtual object. The equation of motion was as follows, defining the target position during frame $f$ as $\mathrm{y}_{f}$ (the position without any visual modification, i.e. the actual hand position in motion-based manipulation), the position of the virtual object as $\mathrm{Y}_{f}$ and the delay parameter as k:

$$
\mathrm{Y}_{f}=\mathrm{Y}_{f-1}+\left(\mathrm{y}_{f}-\mathrm{Y}_{f-1}\right) \cdot \mathrm{k}
$$

With this technique, the virtual object moves slower when the distance between the target position and the current virtual object's position is small and moves faster when the distance is large. Consequently, the virtual object moves slower at the beginning and faster as the distance becomes larger and again slower at the end. It is worth noting that the delay parameter $\mathrm{k}$ works similarly to control/display (c/d) ratio, in that the virtual box moves slower as $\mathrm{k}$ gets smaller, and it does not move at all at if $\mathrm{k}=0$.

Hirao et al. had confirmed this delay parameter method was able to present more levels of sense of weight (4 levels) than changing $\mathrm{c} / \mathrm{d}$ ratio (3 levels). In their experiment, participants lifted a virtual dumbbell under several c/d ratios and delay parameters. The participants compared the experienced pseudo-weight of each with that of default condition where there was no visual modification (that is, the $\mathrm{c} / \mathrm{d}$ ratio equaled to 1 ). Then, the participants rated the experienced pseudo-weight. It was statistically confirmed that the delay parameter technique could present more levels of sense of weight (4 levels) than the $\mathrm{c} / \mathrm{d}$ ratio technique (3 levels). Thus, in order to achieve maximum rating fidelity, we decided to use their technique to compare motion-based versus controller-based pseudo-haptic weight sensations.

According to Hirao et al. [17], participants could distinguish pseudo-weight sensations between the following 4 delay parameter values: $1,0.01-0.005,0.002-0.001,0.0005$. Before our first experiment, we conducted a pilot experiment with 3 participants (including two of the authors) to determine the delay parameters to be used in the actual experiment. In the pilot experiment, the participants compared pseudo-weight sensations of several parameters including those of Hirao et al. [17], both with motionbased and controller-based manipulation. As a result, we decided to use 7 delay parameters: $1,0.005,0.002,0.001,0.0005,0.0002$, and 0 . All of the 3 pilot participants could distinguish the pseudoweight sensations of the parameters in at least one manipulation condition. Moreover, we decided to also use value 0 , because we wanted to see whether there was a difference between a situation where the virtual object moved very slowly and a situation where the virtual object did not move at all.

\subsection{Measurements}

The evaluation was carried out by using a Visual Analog Scale (VAS) on pseudo-haptic weight sensations, and a 7-point rating scale on the unnaturalness of pulling interaction, and an unstructured interview. Participants answered the VAS and rating scale questions inside the VR application after each trial. 
Participants answered the question, "How much weight did you feel by pulling the virtual box?", using a VAS ranging between "No sense of weight: 0 " and "Maximum weight that can be experienced: 100 ". The instructions were as follows: "If you felt a sense of weight as a haptic experience, and not just by rationalizing, please mark how much weight it was with your intuition on the line. The right end of the line represents the maximum weight that can be experienced. Also, if you did not feel a sense of weight as a haptic experience, please mark on the left end, which represents 'No sense of weight'.".

Participants indicated the experienced unnaturalness by answering the question, "How much unnaturalness did you feel from the pulling interaction?", with a 7-points rating scale from "no unnaturalness" to "strong unnaturalness".

\subsection{Procedure}

The participants were 10 university students ( 7 males, 3 females) in their twenties. At the beginning of the experiment, the participants checked the position of the analog stick and the front trigger of the VR controller, and then put on the HMD and held the VR controller with their right hand. Then the participants adjusted their standing position so that the start point of Fig. 2 was to their front right. After the standing position adjustment, the VR scene was changed to the motion-based manipulation condition, and the participants confirmed that the hand model followed their right hand and that the hand could be closed and opened with the VR controller's trigger.

After confirming that the VR scene worked as intended, the participants received an explanation of the experiment task. They then performed the task of pulling the virtual box $(\mathrm{k}=1)$ towards their body and answered the questionnaires of VAS and rating scales in VR space as a practice trial. When they practiced the pulling task, they were instructed to pull the object as soon as possible and with the same velocity in each trial. They repeated a similar practice trial under the controller-based manipulation condition. Then the participants removed the HMD and took a little break. After that, the experiment was conducted according to the following flow:

1. The participants manipulated the hand model freely for one minute for each condition (controller-based and motionbased) in order to get used to the manipulation.

2. One of the manipulations was chosen in random order and the participants freely manipulated the hand model for 10 seconds under that manipulation condition.

3. The participants performed the pulling task under one of the randomly ordered parameter k-conditions with the manipulation from step 2 .

4. After five seconds from the start of pulling, the participants released the hand from the handle with a signal of an experimenter.

5. The participants answered the questionnaires in VR.

The participants iterated the above process from step 2 to step 5, performing each of the 14 conditions ( 2 manipulation conditions and 7 parameter conditions) twice. After the 28 trials, the participants answered the unstructured interview.

\subsection{Results}

The VAS results about the sense of weight are shown in Fig. 3 and the results of unnaturalness are shown in Fig. 4.

Two-factor analysis of variance was performed for both results. Regarding the sense of weight ratings, a significant main effect was observed in the delay parameter $\mathrm{k}(\mathrm{F}(6,114)=31.45, \mathrm{p}<0.001)$. Bonferroni post-hoc tests showed that parameter 1 resulted in a lower sense of weight than other parameters; parameter 0.005 was

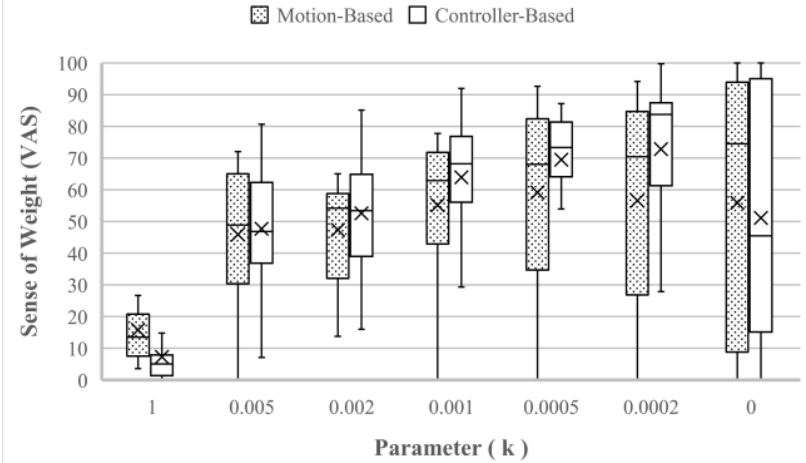

Figure 3: Boxplot of the result of VAS on sense of weight. Boxplots show the median, $25^{\text {th }}$ and $75^{\text {th }}$ percentiles; error bar indicates minima/maxima and cross indicates mean.

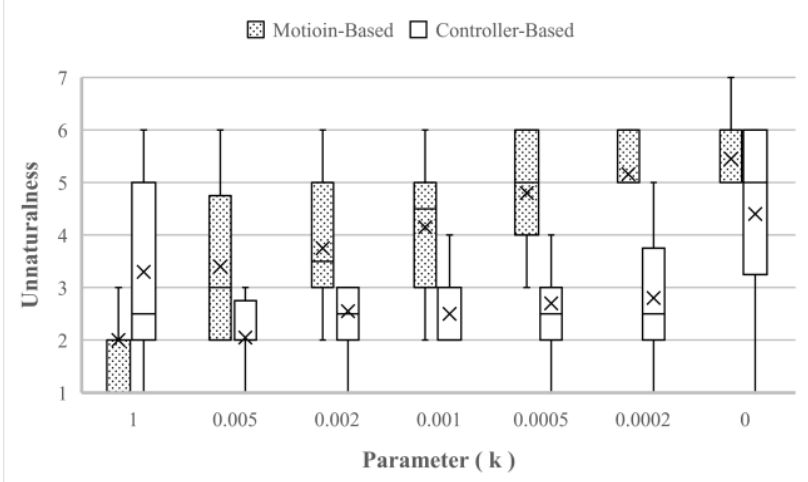

Figure 4: Boxplot of unnaturalness ratings.

Note: the max speed in controller-based manipulation was $2 \mathrm{~m} / \mathrm{s}$

lower than 0.0005 and 0.0002 ; and parameter 0.002 was lower than 0.001 and 0.0005 .

On the other hand, regarding unnaturalness, a significant main effect was observed in both the manipulation condition $(F(1,19)=$ $54.29, \mathrm{p}<0.001)$ and the delay parameter $\mathrm{k}(\mathrm{F}(6,114)=11.55, \mathrm{p}<$ $0.001)$, and a significant interaction between the manipulation condition and the delay parameter $\mathrm{k}$ was also found $(\mathrm{F}(6,114)=$ $17.08, \mathrm{p}<0.001)$.

\subsection{Discussion}

We would like to note that the first experiment had only 10 participants, so the results can only be considered as a tendency and not as strong evidence. The second experiment was performed to provide further details.

Most importantly, the results of our first experiment indicate that controller-based manipulation can present pseudo-weight sensations in a similar way to the motion-based manipulation (Fig. 3 ). It also seems that the variances in the controller-based condition are smaller than that of the motion-based, especially in the slower motion (heavier) condition (excluding $\mathrm{k}=0$ ). Moreover, when inspecting the unnaturalness results (Fig. 4), it seems that the unnaturalness ratings rise as the motion becomes slower in the motion-based condition, but the ratings stay relatively low and stable in the controller-based condition.

Regarding the motion-based condition, there is a tendency that the unnaturalness ratings and variances of sense of weight become larger as the virtual box moves slower, and some participants feel no sense of weight with small values of delay parameter k (Fig. 3). This tendency has also been confirmed in previous research on pseudo-haptics $[15,17,19]$. 
8 out of 10 participants commented that they felt unnatural with small values of $\mathrm{k}$ when using the motion-based manipulation because their actual hand's position and virtual position were quite different. Conversely, they commented that the small values of $\mathrm{k}$ did not bother them when using the controller-based manipulation. Furthermore, 2 out of the 8 aforementioned participants also commented that they had a feeling that their bodies were augmented; that is, they felt as if the hand model was connected to their body with an invisible arm in the controller-based manipulation. These comments suggest that sense of agency and sense of ownership are not broken with the controller-based manipulation even at small values of delay parameter $\mathrm{k}$.

Additionally, some participants commented that the reaction force from the analog stick enhanced the pseudo-haptic weight sensation. This indicates that even physically incorrect haptic feedback can improve the pseudo-haptic experience.

One thing to note is that the controller-based manipulation was rated to have stronger unnaturalness in the condition where the delay parameter $\mathrm{k}$ was 1 . In this condition, the virtual box followed the target position without delay. This means that the virtual box was pulled towards participants at a velocity of $2 \mathrm{~m} / \mathrm{s}$ when tilting the stick backward at the utmost tilt angle. It appears that this too fast pulling experience induced relatively high unnaturalness.

In conclusion, we summarize the results of the first experiment:

- The controller-based manipulation can present pseudo-weight sensations in a similar way to the motion-based manipulation.

- When juxtaposed with motion-based manipulation, the controller-based manipulation has less unnaturalness and smaller variances of pseudo-haptic weight experience.

After the first experiment, we conducted another experiment to compare the controller-based and motion-based pseudo-haptic weight sensations in more detail.

\section{SECOND EXPERIMENT}

In this experiment, participants lifted various weights and adjusted $\mathrm{c} / \mathrm{d}$ ratio in order to match their visual experience with the weight they felt. This is contrary to conventional experiments about pseudo-haptic weight sensation, where participants adjust weights as a response to different $\mathrm{c} / \mathrm{d}$ ratio conditions $[14,16]$. To the best of our knowledge, this was the first experiment to utilize such a reverse adjustment approach for pseudo-haptic weight sensations.

The main objectives of this experiment were 1) to conduct a more detailed comparison of the controller-based and motion-based pseudo-haptic weight and 2) to evaluate whether the adjustment of c/d ratio by participants is a sensible approach for measuring a sense of weight, as opposed to adjusting weights.

\subsection{Equipment and Stimuli}

The experimental environment is shown in Fig. 5. The experiment participants were seated and held right and left VR controllers (Oculus Touch) with each hand. The right controller had a bag attached to it so that the controller's total weight was adjustable by placing weight units inside the bag. The total weight of the controller and the empty bag was $180 \mathrm{~g}$. In the experiment, we used 6 controller weight conditions with total weights of $280 \mathrm{~g}, 300 \mathrm{~g}$, $480 \mathrm{~g}, 680 \mathrm{~g}, 880 \mathrm{~g}$, and $1080 \mathrm{~g}$. We chose these values because we wanted to include a weight surpassing $1 \mathrm{~kg}$ (1080g condition), which is obviously heavy compared to the $280 \mathrm{~g}$ but not too heavy for participants to be exhausted during the task. Additionally, we wanted to include one condition ( $300 \mathrm{~g}$ condition) that differed very little from the lightest $280 \mathrm{~g}$ condition, because certain previous studies $[14,16]$ indicated that pseudo-haptic weight sensations resulted in very small - only around $5 \%$ from the default weight. During the experiment, the participants wore a VR HMD (Oculus

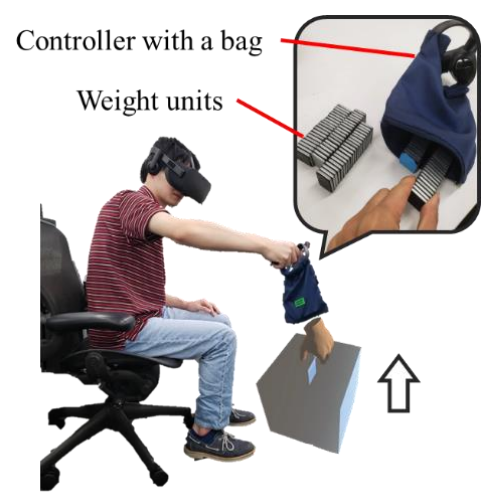

Figure 5: Experimental environment (motion-based manipulation)

Rift CV1). In the VR environment, a virtual box was on the floor to the front right of the participants.

The manipulation task was very close to that of the first experiment. In this experiment, the participants lifted the virtual object up instead of pulling it. In the motion-based manipulation, the position and rotation of a right-hand model followed the pose of the right-hand VR controller. In this manipulation, the participants extended their actual right hand to reach the handle of the virtual box, pressed the right-hand controller's front trigger to grab it, and lifted their actual hand.

In the controller-based manipulation, the hand model stayed in the air and had no connection to the movement of the actual hand. By tilting the right-hand controller's analog stick forward or backward, the participants were able to move the hand model along a vertical line segment from a start point at the participants' stomach height to an end point at the virtual handle location that was $0.5 \mathrm{~m}$ under the start point.

In this experiment, we set the top velocity of the virtual hand in the controller-based manipulation to $1.5 \mathrm{~m} / \mathrm{s}$. This velocity was based on the results of a pilot experiment, where 3 participants adjusted the top velocity until it felt natural.

The experimental task in the controller-based manipulation condition was to tilt the right-hand controller's analog stick forward to move the hand model down from the start point to the handle position, press the right-hand controller trigger to grab the handle, and then tilt the analog stick backward to lift the virtual box.

In both manipulation conditions, the handle (and the virtual box) were constrained to move vertically from the end point to the start point during the grabbing phase. When not grabbing the handle, the $\mathrm{c} / \mathrm{d}$ ratio remained at a value of 1 in both manipulations.

In this experiment, we utilized the variable $\mathrm{c} / \mathrm{d}$ ratio technique to induce pseudo-haptic weight sensation. We used this technique because it is utilized by most of prior studies that measure pseudoweight sensations by physical weight. Consequently, the results of the second experiment are easier to compare with the results from this prior research. The equation of motion in the $\mathrm{c} / \mathrm{d}$ ratio technique is as follows, defining the target position during frame $f$ as $\mathrm{y}_{f}$, the position of the virtual object as $\mathrm{Y}_{f}$ and the c/d ratio as cd:

$$
\mathrm{Y}_{f}=\mathrm{Y}_{0}+\left(\mathrm{y}_{f}-\mathrm{Y}_{0}\right) \cdot \mathrm{cd}
$$

\subsection{Measurements}

We chose the unconventional approach of letting participants rate pseudo-haptic weight by adjusting c/d ratios in different weight conditions instead of vice versa because of two reasons. 1) Fast adjustment of the controller's physical weight with sufficient fidelity is difficult to implement. 2) We wanted to go beyond the typical experimental setting, where the weight adjustments are limited to small deviations from the base weight, either due to the 
weak motor of the chosen haptic device or due to experimenter choice. Some studies have suggested that the $\mathrm{c} / \mathrm{d}$ ratio technique can at best achieve a pseudo-weight that is only around 5\% heavier from the physical weight $[14,16]$. However, this is not supported by other studies that rely on subjective evaluations $[15,17]$ nor by our first experiment. Moreover, the optimal minimum step of weight adjustment is unknown.

The adjustable range of c/d ratio was from 0.01 to 2 and the minimum step of the adjustment was 0.01 . Participants were able to adjust c/d ratio by tilting the left-hand controller's analog stick to the left (decrease) or right (increase). Besides adjusting the $\mathrm{c} / \mathrm{d}$ ratio, the left-hand controller had no other function in this experiment.

\subsection{Procedure}

The participants were 20 university students ( 17 males, 3 females) in their twenties. Upon beginning the experiment, the participants wore the HMD and held right and left VR controllers with respective hands. First, the participants were instructed on how to manipulate the virtual hand with the controller-based and motionbased manipulations. After confirming that the participants had understood both manipulation approaches, they received an explanation about the experiment task. They were instructed to express out loud during the experiment if they ever felt that there was no $\mathrm{c} / \mathrm{d}$ ratio that would have matched the target weight. Then the participants practiced performing the task (below steps from 1 to 5) for each manipulation under two different weight conditions: $280 \mathrm{~g}$ and $1080 \mathrm{~g}$. This was followed by a short break, after which the experiment was conducted according to the following flow:

1. The participants established the target weight experience by lifting the virtual box using the motion-based manipulation with a c/d ratio of 1.0, while the right controller's weight was one of the following weight conditions encountered in a random order: $280 \mathrm{~g}, 300 \mathrm{~g}, 480 \mathrm{~g}, 680 \mathrm{~g}, 880 \mathrm{~g}$, and $1080 \mathrm{~g}$. The participants were asked to remember the weight sensation.

2. The manipulation condition was set to either controller-based or motion-based and the initial $\mathrm{c} / \mathrm{d}$ ratio was set to either 0.01 or 2.0 from the remaining randomly ordered trial permutations. The right-hand controller's weight was then reset to $280 \mathrm{~g}$.

3 . The participants lifted the virtual box under the chosen manipulation condition using the right-hand controller.

4. The participants adjusted the $\mathrm{c} / \mathrm{d}$ ratio with the left-hand controller.

5. The participants repeated step 3 and step 4 until they felt that the weight sensation in step 3 equaled to that of their recollection from step 1 .

The participants repeated steps 1 to 5 for 24 randomly ordered conditions ( 2 manipulation conditions, 6 weight conditions, and 2 initial $\mathrm{c} / \mathrm{d}$ ratio conditions).

\subsection{Results}

The result of the adjusted c/d ratio is shown in Fig. 6. For each participant we averaged the $\mathrm{c} / \mathrm{d}$ ratio of both initial $\mathrm{c} / \mathrm{d}$ ratio conditions, yielding $12 \mathrm{c} / \mathrm{d}$ ratio data points per participant. Every participant managed to adjust all of the $\mathrm{c} / \mathrm{d}$ ratio trials.

Two-factor analysis of variance was performed. As a result, a significant main effect was observed in both controller's weight condition $(\mathrm{F}(5,95)=138.8, \mathrm{p}<0.001)$ and manipulation condition $(\mathrm{F}(1,19)=53.77, \mathrm{p}<0.001)$. Bonferroni post-hoc tests showed that all pairs of adjusted c/d ratios of a physical weight condition are significantly different excluding the pair of $280 \mathrm{~g}$ and $300 \mathrm{~g}$ conditions. Fig. 7 and Fig. 8 show the approximate curve of the adjusted $\mathrm{c} / \mathrm{d}$ ratio in both manipulation conditions.

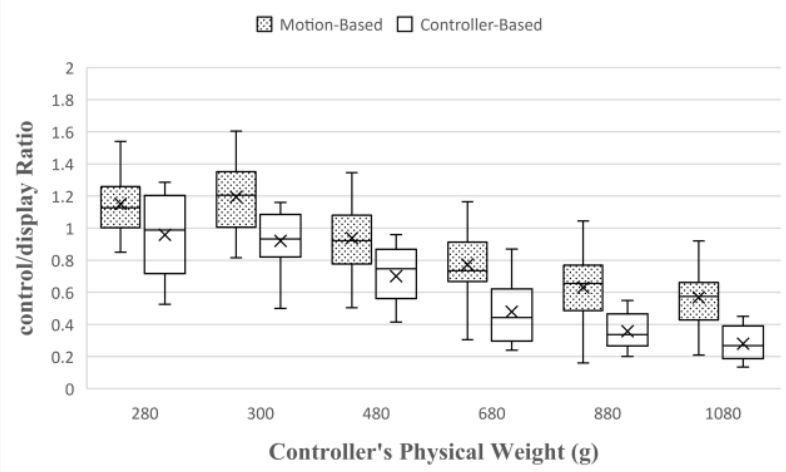

Figure 6: Boxplot of the result of the control/display ratio.

Note: the max speed in controller-based manipulation was $1.5 \mathrm{~m} / \mathrm{s}$.

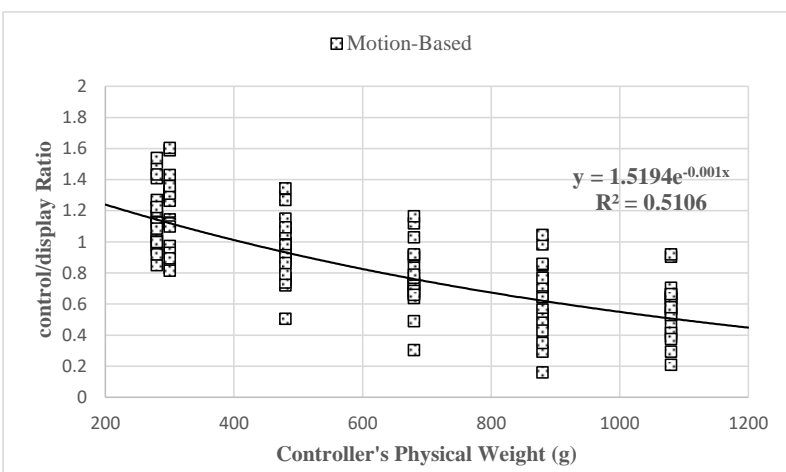

Figure 7: Approximate curve of the averages of the adjusted control/display ratio in motion-based condition.

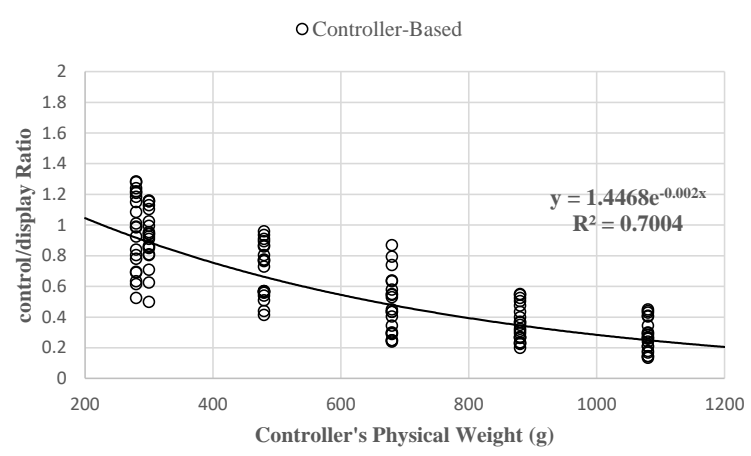

Figure 8: Approximate curve of the averages of the adjusted control/display ratio in controller-based condition.

Note: the max speed in controller-based manipulation was $1.5 \mathrm{~m} / \mathrm{s}$.

\subsection{Discussion}

The results suggest that our approach of adjusting the $\mathrm{c} / \mathrm{d}$ ratio for measuring a sense of weight is useful, because for both manipulations the adjusted c/d ratio values become smaller as the physical weight gets heavier and their variances are relatively tightly bounded (Fig 6.).

Upon further examining Fig. 6, we can see that the results reflect those of the first experiment (Fig. 3): the controller-based manipulation can present pseudo-haptic weight in a consistent fashion that closely resembles the conventional motion-based manipulation with regard to the adjusted c/d ratio values and the associated physical weight.

In this experiment, our results differ from those of prior studies $[14,16]$, which measured pseudo-weight sensations by adjusting 
the physical weight. The main differences between our experiment and theirs are the adjustment method and the method of comparing the sense of weight. In their experiment, the participants had one object for each hand and adjusted the physical weight of one of the objects until they felt that the two objects' weights were equal. In such setups, the haptic (physical weight) information becomes emphasized in the cross-modal integration because the participants feel and compare the two objects' physical weights simultaneously. On the other hand, in our experiment the participants must first memorize the physical weight and then recollect that memory while adjusting the visual motion of the virtual box. We speculate that in our approach the participants focus more on the visual information when adjusting the weight sensation. These differences in the experimental designs can affect the pseudo-haptic experience, which in turn could lead to different results. Nevertheless, we argue that our experimental design of adjusting $\mathrm{c} / \mathrm{d}$ ratio is in some sense more grounded to the practical reality of current consumer VR hardware (which lacks grounded force feedback devices), while also being exclusively focused on purely virtual, pseudo-haptic sensations as opposed to focusing on real physical changes.

\section{Conclusion}

In this paper, we compared controller-based versus motion-based pseudo-haptic weight sensations in VR. This was done in experiments where participants manipulated a virtual hand both with a game controller's analog stick and with a motion controller, and compared the pseudo-haptic weight sensations of both approaches. The results show that the controller-based manipulation was able to present pseudo-weight sensations in a similar way to the motion-based manipulation.

Our study suggests that VR developers and users can utilize the controller-based manipulation to present a pseudo-haptic experience as an alternative to the conventional motion-based approach. Our research is limited by the restriction of moving the virtual hand only along one axis during drawing/lifting the virtual object in the experiments and because an analog stick provides only 2D input. Therefore, the results could be different if these pseudoweight techniques are used for 3D movements. Moreover, we do not present a method for manipulating a whole full-body virtual avatar by a game controller. The choice of which physical body part to manipulate and how to manipulate could affect the pseudo-haptic experience. In future research, we will explore the relationship between different manipulation techniques and pseudo-haptic experiences, and explore controller-based interaction systems for manipulating full body avatars.

\section{References}

[1] M. A. Srinivasan and C. Basdogan, "Haptics in Virtual Environments: Taxonomy, Research Status, and Challenges," Computers and Graphics, vol.21, no.4 (1997) pp.393-404.

[2] T. H. Massie and J. K. Salisbury, "PHANToM haptic interface: a device for probing virtual objects", American Society of Mechanical Engineers, Dynamic Systems and Control Division (Publication) DSC, Vol.55-1 (1994) pp.295-299.

[3] M. Sato, "SPIDAR and virtual reality", Multimedia, Image Processing, and Soft Computing: Trends, Principles and Applications Proceedings of the 5th Biannual World Automation Congress, WAC 2002, ISSCI 2002 and IFMIP 2002, vol.13 (2002) pp.17-23.

[4] A. Zenner and A. Kruger, "Shifty: A Weight-Shifting Dynamic Passive Haptic Proxy to Enhance Object Perception in Virtual Reality," IEEE Transactions on Visualization and Computer Graphics 23, 4 (2017), pp.1285-1294.

[5] J. Shigeyama, T. Hashimoto, S. Yoshida, T. Narumi, T. Tanikawa, and M. Hirose, "Transcalibur: A Weight Shifting Virtual Reality Controller for 2D Shape Rendering based on Computational
Perception Model," in Proceedings of the 2019 CHI Conference on Human Factors in Computing Systems (CHI'19), ACM, (2019).

[6] S. Sagheb, F. W. Liu, A. Bahremand, A. Kidane, and R. Likamwa, "SWISH: A Shifting-Weight Interface of Simulated Hydrodynamics for Haptic Perception of Virtual Fluid Vessels," in Proceedings of the $32^{\text {nd }}$ Annual ACM Symposium on User Interface Software and Technology (UIST'19), ACM, (2019), pp.751-761.

[7] A. Zenner and A. Kruger, "Drag:on: A Virtual Reality Controller Providing Haptic Feedback Based on Drag and Weight Shift," in Proceedings of the 2019 CHI Conference on Human Factors in Computing System (CHI'19), ACM, (2019).

[8] S. Heo, C. Chung, G. Lee, and D. Wigdor, "Thor's Hammer: An Ungrounded Force Feedback Device Utilizing Propeller-Induced Propulsive Force," in Extended Abstracts of the 2018 CHI Conference on Human Factors in Computing Systems (CHI EA'18), ACM, (2018).

[9] K. Minamizawa et al., S. Tachi, "Gravity grabber: Wearable haptic display to present virtual mass sensation" ACM SIGGRAPH'7 Article number 8, (2007).

[10] T. Mitsuda, "Pseudo Force Display by the Use of Constrictive Pressure on a Wrist," The Virtual Reality Society of Japan, Vol. 12, No. 4 (2007), pp.577-584 (in Japanese)

[11] T. Amemiya, T. Maeda, "Asymmetric Oscillation Distorts the Perceived Heaviness of Handheld Objects", IEEE Transactions on Haptics, Vol. 1, Issue 1 (2008), pp.9-18.

[12] A. Lecuyer, "Simulating haptic feedback using vision: A survey of research and applications of pseudo-haptic feedback," Presence: Teleoperators and Virtual Environments, vol. 18 (2009), pp. 39-53.

[13] L. Dominjon, A. Lecuyer, J. M. Burkhardt, P. Richard, and S. Richir, "Influence of control/display ratio on the perception of mass of manipulated objects in virtual environments," IEEE Proceedings. VR 2005. Virtual Reality, (2005), pp.19-25.

[14] Y. Taima, Y. Ban, T. Narumi, T. Tanikawa and M. Hirose, "Controlling fatigue while lifting objects using Pseudo-haptics in a mixed reality space," 2014 IEEE Haptics Symposium (HAPTICS, Houston, TX, USA, 2014) pp.175-180.

[15] M. Rietzler, F. Geiselhart, J. Gugenheimer, and E. Rukzio, "Breaking the Tracking: Enabling Weight Perception using Perceivable Tracking Offsets," CHI '18 Proceedings of the 2018 CHI Conference on Human Factors in Computing Systems, Paper No. 128 (2018).

[16] M. Samad, E. Gatti, A. Hermes, H. Benko, and C. Parise, "PseudoHaptic Weight: Changing the Perceived Weight of Virtual Objects By Manipulating Control-Display Ratio," Proceedings of the 2019 CHI Conference on Human Factors in Computing Systems, Paper No. 320 (2019).

[17] Y. Hirao, R. Mitsuya and T. Kawai, "Weight Sense Representation Using Cross-modality in Virtual Reality," The Virtual Reality Society of Japan, Vol.23, No.4 (2018), (in Japanese).

[18] L. Lin, A. Normoyle, A. Adkins, Y. Sun, A. Robb, Y. Ye, M. D. Luca, and S. Jorg, "The Effect of Hand Size and Interaction Modality on the Virtual Hand Illusion," in Proceedings of 2019 IEEE Conference on Virtual Reality and 3d User Interfaces (VR), (2019).

[19] A. Pusch and A. Lecuyer, "Pseudo-haptics: from the theoretical foundations to practical system design guidelines," in Proceedings of ICMI 2011 (2011), pp. 57-64

[20] K. Ma and B. Hommel, "Body-ownership for actively operated noncorporeal objects," Consciousness and Cognition, Vol. 36 (2015), pp.75-86

[21] B. E. Insko, "Passive Haptics Significantly Enhances Virtual Environments," Ph.D. Dissertation, The University of North Carolina at Chapel Hill, Advisor(s) F. P. Brooks, Jr, (2001).

[22] A. L. Simeone, E. Velloso, and H. Gellersen, "Substitutional Reality: Using the Physical Environment to Design Virtual Reality Experiences," in Proceedings of the 33rd Annual ACM Conference on Human Factors in Computing Systems (CHI'15), ACM, (2015), pp.3307-3316. 